\title{
A study of particle paths in non-axisymmetric Taylor-Couette flows
}

\author{
By P. ASHWIN ${ }^{1} \dagger$ AND G. P. KING ${ }^{2}$ \\ ${ }^{1}$ Institut Non Linéaire de Nice, 1361 Route des Lucioles, 06560 Valbonne, France \\ ${ }^{2}$ Mathematics Institute and Department of Engineering, \\ University of Warwick, Coventry CV4 7AL, UK
}

(Received 1 March 1996 and in revised form 8 November 1996)

We study the paths of fluid particles in velocity fields modelling rigidly rotating velocity fields that occur in the concentric Taylor problem. We set up velocity fields using the model of Davey, DiPrima \& Stuart (1968) based on small-gap asymptotics. This allows a numerical study of the Lagrangian properties of steady flow patterns in a rotating frame. The spiral and Taylor vortex modes are integrable, implying that in these cases almost all particle paths are confined to two-dimensional surfaces in the fluid. For the case of Taylor vortices the motion on these surfaces is quasi-periodic, whereas for spirals the particles propagate up or down the cylinder on these surfaces.

The non-axisymmetric modes we consider are wavy vortices, spirals, ribbons and twisted Taylor vortices. All of these flows have the property that they are steady flows when examined in a rotating frame of reference. For all non-axisymmetric modes with the exception of spirals, we observe the existence of regions of chaotic mixing within the fluid. We discuss mixing of the fluid by these flows with reference to the pattern of stagnation points and some of the periodic trajectories within the fluid and on the boundary.

\section{Introduction}

Little is at present understood about the geometrical structure of fully threedimensional steady and unsteady fluid flows and even less about the physics of chaotic advection in these flows. In order to further our understanding there is a need to explore the kinematic behaviour of fluid particles in laminar, fully threedimensional solutions of the Navier-Stokes equations. In an earlier paper (Ashwin \& King 1995) we investigated the particle paths given by DiPrima \& Stuart's (1975) asymptotic solution for Taylor vortex flow between eccentric cylinders. In this paper we investigate the particle paths given by the Davey, DiPrima \& Stuart (1968) model for non-axisymmetric flows between concentric rotating cylinders.

The Taylor-Couette apparatus has provided much insight into and a test-bed for theories of incompressible fluid flows. Due to its simple symmetric form (two axisymmetric rotating cylinders with a fluid between them) it has been used as a paradigm for breakdown of laminar to turbulent flow. Many of the flow patterns can be classified according to their symmetries; see, for example, Chossat \& Iooss (1994). On rotating the inner cylinder while keeping the outer cylinder stationary,

$\dagger$ Permanent address: Department of Mathematical and Computing Sciences, University of Surrey, Guildford GU2 5XH, UK. 
Couette flow (consisting of two-dimensional flow in planes perpendicular to the axis) becomes unstable to motion in the axial direction; this gives rise to Taylor vortex flow at a critical value of the Taylor number. Increasing the Taylor number further gives rise to secondary instabilities in the form of wavy vortex flow where a wave-like disturbance propagates around the Taylor vortices. Rotating both cylinders can give rise to a variety of other non-axisymmetric flows, for example spirals, ribbons and twisted vortices, see e.g. Andereck, Liu \& Swinney (1986).

The majority of investigations into flow patterns in the Taylor-Couette apparatus have focused on the Eulerian properties of the flow. However, the Lagrangian properties, necessary to understand the mixing properties of the flow, are often not easily deducible from the Eulerian velocity field.

In Couette flow the constraints of two dimensions and rotational symmetry restrict the particle paths to closed orbits. The rotational symmetry of three-dimensional Taylor vortex flow means that it is effectively an integrable flow and almost all particle paths are quasi-periodic. On breaking the continuous rotational symmetry of the flow pattern, this reason for integrability is removed and we expect a nonintegrable flow pattern with a certain degree of chaotic mixing. Two ways in which the rotational symmetry of the flow pattern can be broken are (i) by perturbing the geometry of the apparatus, and (ii) by non-axisymmetric perturbations of the flow field. The former case was studied in Ashwin \& King (1995). After ensuring preservation of volume, chaotic layers of mixing were found for small and large eccentricities. At large eccentricities, stagnation points appeared in the skin friction field on the outer cylinder and stronger mixing was observed, apparently related to the presence of these points.

Broomhead \& Ryrie (1988) investigated the effect of breaking the symmetry of the flow field using a kinematic model consisting of a volume-preserving flow that has some basic features of wavy vortex flow, namely a Taylor vortex-type flow superimposed with an oscillation in the axial direction. They found evidence for chaotic layers of mixing, especially in the region near the cylinders and the inflow and outflow boundaries. However, their model has only a qualitative resemblance to a solution of the Navier-Stokes equations. As discussed in Ashwin, Mann \& King (1995), this means that they missed the possibility of the presence of ring vortices in wavy vortex flow propagating azimuthally around the cylinder. We interpret the presence of such ring vortices encircling the centres of the vortices as being indicative of the same sort of mechanisms being present here as in vortex breakdown (for example, Leibovich 1978; Brown \& Lopez 1990).

In order to investigate particle paths in wavy vortex and associated flows, we consider an asymptotic model of Davey et al. (1968) giving the velocity field near the onset of the wavy vortex instability. This uses assumptions of a small gap between the cylinders and closeness to criticality so that a weakly nonlinear theory can be applied. The asymptotics are in terms of a small parameter $\delta=d / R_{0}$ where $d$ is the gap and $R_{0}$ is the mean radius of the two cylinders.

Section 2 introduces the necessary notation and details of the model. Section 3 briefly reviews some results from the weakly nonlinear theory, while $\$ 4$ examines the resulting particle paths for various steady flow patterns associated with instability of Couette flow. These are interpreted with the help of the 'flow skeleton' (Perry \& Chong 1987; MacKay 1994). In particular, the robustness of the obtained flow skeletons to perturbations respecting the symmetries of the flow patterns lends support to the hypothesis that the particle dynamics of the first-order flow are modelling those in the true flow. Finally, in $\S 5$ we discuss the effects of the observed mixing in the 
flow patterns. We contrast the observed axial transport mechanisms in wavy vortex and spiral flow. In agreement with a diffusion model we find linear growth of the variance of an ensemble of particles near the boundaries of a wavy vortex, whereas axial dispersion in spiral flow is typical of non-chaotic flows.

\section{The model and scalings}

Following the notation of Davey et al. (1968) we consider the flow between two cylinders of infinite length and radii $R_{1}$ and $R_{2}>R_{1}$ rotating about their common axis; we set

$$
R_{0}=\frac{R_{1}+R_{2}}{2}, \quad d=R_{2}-R_{1}, \quad \eta=\frac{R_{1}}{R_{2}} \text { and } \delta=d / R_{0} .
$$

Consider the inner cylinder rotating with angular velocity $\Omega_{1}$, the outer with $\Omega_{2}$. Set

$$
\Omega_{0}=\frac{\Omega_{1}+\Omega_{2}}{2}, \quad \alpha=2\left(\frac{1-\mu}{1+\mu}\right) \quad \text { and } \mu=\frac{\Omega_{2}}{\Omega_{1}} .
$$

Let $(R, \theta, Z)$ be cylindrical polar coordinates (the $Z$-axis is the common axis of the cylinders) and $\left(u_{R}, u_{\theta}, u_{Z}\right)$ the velocity components of the fluid in these directions. The well-known Couette solution of the incompressible Navier-Stokes equations for a Newtonian fluid with kinematic viscosity $v$ in this geometry is given by

$$
u_{R}=u_{Z}=0, \quad u_{\theta}=V(R)=A R+\frac{B}{R}
$$

where $A$ and $B$ are the constants

$$
\begin{array}{r}
A=\frac{R_{2}^{2} \Omega_{2}-R_{1}^{2} \Omega_{1}}{R_{2}^{2}-R_{1}^{2}}=-\Omega_{1} \frac{\eta^{2}-\mu}{1-\eta^{2}}, \\
B=-\frac{R_{1}^{2} R_{2}^{2}\left(\Omega_{2}-\Omega_{1}\right)}{R_{2}^{2}-R_{1}^{2}}=\Omega_{1} R_{1}^{2} \frac{1-\mu}{1-\eta^{2}} .
\end{array}
$$

We consider perturbations $\left(u^{\prime}, v^{\prime}, w^{\prime}\right)$ from Couette flow. We scale the variables by introducing dimensionless $x, \phi, \zeta$ and $\tau$ :

$$
R=R_{0}+x d, \quad Z=\zeta d, \quad \theta=\frac{\Omega_{0} d^{2}}{v} \phi, \quad t=\frac{d^{2}}{v} \tau ;
$$

and scale the narrow-gap limit of the Couette flow velocity $V(R)$ and the velocity perturbations $\left(u^{\prime}, v^{\prime}, w^{\prime}\right)$ to give dimensionless $(u, v, w)$ :

$$
V(x)=R_{0} \Omega_{0} \Omega_{l}(x), \quad u^{\prime}=-\frac{v}{\alpha d} u, \quad v^{\prime}=R_{0} \Omega_{0} v, \quad w^{\prime}=-\frac{v}{\alpha d} w,
$$

where

$$
\Omega_{l}(x)=1-\alpha x .
$$

We introduce the dimensionless Taylor number:

$$
T=-\frac{4 A \Omega_{0} d^{4}}{v^{2}} .
$$

It is convenient to scale $\zeta$ further by a constant $\lambda$ corresponding to the scaled axial wavelength; i.e. we define

$$
z=\lambda \zeta=\frac{\lambda}{d} Z
$$


The particle paths for the original velocity field $\left(u_{R}, u_{\theta}, u_{Z}\right)$ are given by integrating the vector field:

$$
\frac{\mathrm{d} R}{\mathrm{~d} t}=u_{R}, \quad R \frac{\mathrm{d} \theta}{\mathrm{d} t}=u_{\theta}, \quad \frac{\mathrm{d} Z}{\mathrm{~d} t}=u_{Z} .
$$

Changing to the coordinates $(x, \theta, z)$ the particle paths are given by solutions of

$$
\frac{\mathrm{d} x}{\mathrm{~d} t}=\frac{1}{d} u_{R}, \quad \frac{\mathrm{d} \theta}{\mathrm{d} t}=\frac{1}{R_{0}(1+\delta x)} u_{\theta}, \quad \frac{\mathrm{d} z}{\mathrm{~d} t}=\frac{\lambda}{d} u_{Z} .
$$

Including the above scalings and writing $\dot{x}=\mathrm{d} x / \mathrm{d} \tau$ we have, on truncating all terms to leading order in $\delta$,

$$
\dot{x}=-\frac{u}{\alpha}, \quad \dot{\theta}=\frac{d^{2} \Omega_{0}}{v}(1-\alpha x+v), \quad \dot{z}=-\frac{\lambda w}{\alpha} .
$$

We will deal exclusively with the case $\Omega_{2}=0$ (outer cylinder stationary) where $A=\Omega_{1} /\left(1-\left(R_{2} / R_{1}\right)^{2}\right)=-\left(\Omega_{0}(2-\delta)^{2}\right) /(4 \delta)$, giving to leading order in $\delta$

$$
\frac{d^{2} \Omega_{0}}{v}=\frac{(T \delta)^{1 / 2}}{2}
$$

and so the equations for particle paths are, to leading order

$$
\dot{x}=-\frac{1}{\alpha} u, \quad \dot{\theta}=\frac{(T \delta)^{1 / 2}}{2}(1-\alpha x+v), \quad \dot{z}=-\frac{\lambda}{\alpha} w .
$$

Note that the flow (2.3) does not exactly preserve a volume element $R \mathrm{~d} R \mathrm{~d} Z \mathrm{~d} \theta$ (even when $u=v=w=0$ ). However, it does exactly preserve an 'approximate' volume element $\mathrm{d} x \mathrm{~d} z \mathrm{~d} \theta$, and thus can give a qualitatively realistic model of the particle paths. This is discussed in more detail in $\$ 2.3$.

\subsection{First-order approximation of the velocity perturbation}

We do not discuss the model of Davey et al. (1968) in detail, nor shall we dwell on its derivation. Basically, it considers $z$-periodic perturbations to the velocity field of Couette flow that break the azimuthal symmetry. By letting $\delta \rightarrow 0$ while holding $\Omega_{0}^{2} R_{0} d^{3} / v^{2}$ and $v \theta / \Omega_{0} d^{2}$ fixed they show that the linear modes of instability can be written using a Fourier series expansion of the scaled perturbations $u, v$ and $w$ in the $z($ period $2 \pi)$ and the $\theta($ period $2 \pi / m)$ directions. For example,

$$
v(x, \theta, z, \tau)=\sum_{q=-\infty}^{\infty}\left[v_{o q}(x, \tau)+\sum_{n=1}^{\infty}\left(v_{c n q}(x, \tau) \cos (n z)+v_{s n q}(x, \tau) \sin (n z)\right)\right] \mathrm{e}^{\mathrm{i} q m \theta} .
$$

They define

$$
k=m \Omega_{0} d^{2} / v,
$$

a scaled azimuthal wavenumber such that $m \theta=k \phi$. Davey et al. restrict their attention to the interaction of the axisymmetric $q=0, n=1$ and the $q=1, n=1$ non-axisymmetric terms. These are found to be the most highly amplified modes close to primary instability in the limit $\delta \rightarrow 0$. 
Restricting to linear modes the equations for $(u, v, w)$ are given by

$$
\left.\begin{array}{rl}
u= & A_{c}(\tau) f_{20}(x) \cos (z)+A_{s}(\tau) g_{20}(x) \sin (z) \\
& +B_{c}(\tau) h_{20}(x) \cos (z) \mathrm{e}^{\mathrm{i} m \theta}+B_{s}(\tau) l_{20}(x) \sin (z) \mathrm{e}^{\mathrm{i} m \theta}+\text { c.c. }, \\
v= & A_{c}(\tau) f_{0}(x) \cos (z)+A_{s}(\tau) g_{0}(x) \sin (z) \\
& +B_{c}(\tau) h_{0}(x) \cos (z) \mathrm{e}^{\mathrm{i} m \theta}+B_{s}(\tau) l_{0}(x) \sin (z) \mathrm{e}^{\mathrm{i} m \theta}+\text { c.c. }, \\
w= & A_{c}(\tau) f_{30}(x) \sin (z)+A_{s}(\tau) g_{30}(x) \cos (z) \\
& +B_{c}(\tau) h_{30}(x) \sin (z) \mathrm{e}^{\mathrm{i} m \theta}+B_{s}(\tau) l_{30}(x) \cos (z) \mathrm{e}^{\mathrm{i} m \theta}+\text { c.c. },
\end{array}\right\}
$$

where c.c. denotes the complex conjugate. There are higher-order corrections in $A_{c}$, $\cdots, B_{s}$, but we ignore these since we are close to the onset of instability. The functions $f_{0}, g_{0}, h_{0}$ and $l_{0}$ are found by solving the following sixth-order system of ordinary differential equations:

$$
\left.\begin{array}{l}
M\left(\lambda, a_{c 0}, 0, T\right) f_{0}=0, \quad M\left(\lambda, a_{s 0}, 0, T\right) g_{0}=0, \\
M\left(\lambda, b_{c 0}, k, T\right) h_{0}=0, \quad M\left(\lambda, b_{s 0}, k, T\right) l_{0}=0,
\end{array}\right\}
$$

where

$$
M\left(\lambda, a_{c 0}, k, T\right)=N\left(\lambda, a_{c 0}, k\right)\left(\mathrm{D}^{2}-\lambda^{2}\right) N\left(\lambda, a_{c 0}, k\right)+\lambda^{2} T \Omega_{l}(x)
$$

and

$$
N\left(\lambda, a_{c 0}, k\right)=\mathrm{D}^{2}-\lambda^{2}-a_{c 0}-\mathrm{i} k \Omega_{l}(x) .
$$

(Note that $\mathrm{D}$ represents $\mathrm{d} / \mathrm{d} x$.) The other components are

$$
\begin{array}{ll}
f_{20}=N\left(\lambda, a_{c 0}, 0\right) f_{0}, & f_{30}=-\lambda^{-1} \mathrm{D} f_{20}, \\
g_{20}=N\left(\lambda, a_{s 0}, 0\right) g_{0}, & g_{30}=\lambda^{-1} \mathrm{D} g_{20}, \\
h_{20}=N\left(\lambda, b_{c 0}, k\right) h_{0}, & h_{30}=\lambda^{-1}\left(-\mathrm{D} h_{20}+\mathrm{i} k \alpha h_{0}\right), \\
l_{20}=N\left(\lambda, b_{s 0}, k\right) l_{0}, & l_{30}=\lambda^{-1}\left(\mathrm{D} l_{20}-\mathrm{i} k \alpha l_{0}\right) .
\end{array}
$$

The boundary conditions for the system (2.6) implied by radial continuity and no-slip boundary conditions are

$$
h_{0}=\mathrm{D}^{2} h_{0}=N\left(\lambda, b_{c 0}, k\right) \mathrm{D} h_{0}=0 \text { at } x= \pm \frac{1}{2}
$$

with similar equations for $f_{0}$ (take $k=0$ and replace $b_{c 0}$ by $a_{c 0}$ ), $g_{0}$ (take $k=0$ and replace $b_{c 0}$ by $a_{s 0}$ ) and $l_{0}$ (with $b_{c 0}$ replaced by $b_{s 0}$ ).

Using the axial translational symmetry of the Couette flow, the following identities can be assumed:

$$
\begin{aligned}
& a_{s 0}=a_{c 0}=a_{0}, \quad b_{s 0}=b_{c 0}=b_{0}, \\
& g_{0}=f_{0}, \quad g_{20}=f_{20}, \quad g_{30}=-f_{30}, \\
& l_{0}=h_{0}, \quad l_{20}=h_{20}, \quad l_{30}=-h_{30} \text {. }
\end{aligned}
$$

\subsection{Solving the boundary value problems}

The boundary value problems (2.6), (2.7) were solved numerically using a Chebyshev collocation method, NAG routine D02TGF. Given values of the parameters $T, \delta$ and $m$ we computed the appropriate values of $a_{0}, b_{0 r}$ and $b_{0 i}$ for non-trivial solutions of the eigenvalue problem. This is achieved by imposing a normalizing boundary condition $f_{0}(0)=1.0$ and searching for $a_{0}$ such that $f_{0}(-0.5)=0.0$.

Exact values of the derivatives of the approximate $f_{0}$ are found using the NAG routine E02AHF and hence we find the value of $f_{0}, f_{20}, f_{30}$ at any position in the gap between the two cylinders corresponding to $x \in[-0.5,0.5]$. A similar procedure is used to find $g_{0}$ and $h_{0}$. 


\subsection{Ensuring volume preservation}

The continuity equation in the original variables can be written

$$
\frac{1}{R} \frac{\partial}{\partial R}\left(R u_{R}\right)+\frac{1}{R} \frac{\partial u_{\theta}}{\partial \theta}+\frac{\partial u_{Z}}{\partial Z}=0 .
$$

Changing variables to $(x, \theta, z)$ using the scaling discussed at the start of this Section and multiplying by a constant, this is equivalent to

$$
\frac{1}{1+\delta x} \frac{\partial}{\partial x}((1+\delta x) u)-\frac{1}{1+\delta x} \frac{\partial v}{\partial \theta}+\lambda \frac{\partial w}{\partial z}=0 .
$$

On restricting to first order (this is justified as we truncate the solution to first order) we obtain

Finally, using identity (2.2) we have

$$
\frac{\partial u}{\partial x}-\frac{\alpha d^{2} \Omega_{0}}{v} \frac{\partial v}{\partial \theta}+\lambda \frac{\partial w}{\partial z}=0
$$

$$
\frac{\partial u}{\partial x}-\frac{\alpha(T \delta)^{1 / 2}}{2} \frac{\partial v}{\partial \theta}+\lambda \frac{\partial w}{\partial z}=0 .
$$

Note that the first-order approximation of the velocity perturbation satisfies this first-order continuity equation exactly (cf. Ashwin \& King 1995 where the first-order approximation is in fact dissipative). Except for the radial dependence we can write down the velocity field as an analytical expression. By using a polynomial basis for solving the radial problem we can ensure that derivatives are exactly represented. This means that we do not have to correct the velocity field in order to see qualitatively correct volume-preserving behaviour of particle paths. More specifically, it means that Poincare recurrence will apply for the approximate flow and we can also expect to see KAM surfaces for the approximating flows. We will not get spurious phenomena such as 'attractors' for the trajectories of fluid particles.

\subsection{Rotating frame of reference}

By going into a frame of reference rotating at exactly the propagation velocity of a steadily propagating flow pattern we can observe it as a time-independent flow. The appropriate angular velocity is $\omega / m$ for waves whose time and azimuthal dependence is $\exp \mathrm{i}(m \theta-\omega \tau)$ (i.e. azimuthal wavenumber $m$ ) and so we define $\dot{\psi}=\dot{\theta}-\omega / m$ (with arbitrary initial conditions for $\psi$ ) and note that the velocity field is a steady flow in the $(x, \psi, z)$ frame of reference.

We introduce, merely for convenience of viewing the resulting trajectories, the coordinates in the co-rotating frame

$$
r_{x}+\mathrm{i} r_{y}=(1.5+x) \mathrm{e}^{\mathrm{i} \psi}
$$

which corresponds to projection perpendicular to the axis of the coordinates in a rotating reference frame, with an exaggeration of the gap between the cylinders.

\section{Steady solutions of the amplitude equations}

By restricting to the interaction of the two most unstable azimuthal modes $(m=0,1)$ Davey et al. reduce the evolution problem to a set of coupled ordinary differential equations using a Galerkin approximation. Truncating this set of ODEs to third 
order in the amplitudes and using the axial and azimuthal symmetries they obtain

$$
\begin{aligned}
\frac{\mathrm{d} A_{c}}{\mathrm{~d} \tau}=a_{0} A_{c} & +a_{1} A_{c}^{3}+a_{1} A_{c} A_{s}^{2}+a_{3} A_{c}\left|B_{c}\right|^{2} \\
& +a_{4} A_{c}\left|B_{s}\right|^{2}+a_{5} A_{s} B_{c} \tilde{B}_{s}+\tilde{a}_{5} A_{c} \tilde{B}_{c} B_{s}+\ldots, \\
\frac{\mathrm{d} A_{s}}{\mathrm{~d} \tau}=a_{0} A_{s} & +a_{1} A_{s}^{3}+a_{1} A_{s} A_{c}^{2}+a_{3} A_{s}\left|B_{s}\right|^{2} \\
& +a_{4} A_{s}\left|B_{c}\right|^{2}+a_{5} A_{c} B_{s} \tilde{B}_{c}+\tilde{a}_{5} A_{s} \tilde{B}_{s} B_{c}+\ldots, \\
\frac{\mathrm{d} B_{c}}{\mathrm{~d} \tau}=b_{0} B_{c} & +b_{1} B_{c}\left|B_{c}\right|^{2}+b_{2} B_{c}\left|B_{s}\right|^{2}+b_{3} B_{c} A_{c}^{2} \\
& +b_{4} B_{c} A_{s}^{2}+\left(b_{3}-b_{4}\right) B_{s} A_{c} A_{s}+\left(b_{1}-b_{2}\right) \tilde{B}_{c} B_{c}^{2}+\ldots, \\
\frac{\mathrm{d} B_{s}}{\mathrm{~d} \tau}=b_{0} B_{s} & +b_{1} B_{s}\left|B_{s}\right|^{2}+b_{2} B_{s}\left|B_{c}\right|^{2}+b_{3} B_{s} A_{s}^{2} \\
& +b_{4} B_{s} A_{c}^{2}+\left(b_{3}-b_{4}\right) B_{c} A_{s} A_{c}+\left(b_{1}-b_{2}\right) \tilde{B}_{s} B_{s}^{2}+\ldots .
\end{aligned}
$$

The general form of these equations can be derived from symmetry considerations alone by considering the dynamics of a $(0,1)$ mode interaction for a system with $\mathbf{O}(2) \times \mathbf{S O}(2)$ symmetry. This forces us to consider a six-dimensional centre manifold (note that amplitudes $A$ are real and $B$ are complex) and the above equations give all possible terms up to third order. For more details of this, see Golubitsky, Stewart \& Schaeffer (1988, Case Study 6) and Chossat \& Iooss (1994).

To approximate the vector fields corresponding to steady states (in the $\psi$-rotating frame) of the Navier-Stokes equations approximated above, we require values of the coefficients $a_{0}, a_{1}, a_{4}, b_{0}, b_{1}$ and $b_{4}$. Approximating these coefficients requires considerable numerical computation (see e.g. Chossat \& Iooss 1994). To simplify this we follow Krueger, Gross \& DiPrima (1966) and approximate values of $a_{1}, a_{4}$ and $b_{1}$ by their limiting values as $k \rightarrow 0$ (assuming they are regular as $\delta \rightarrow 0$ ). The values of $b_{4}$ we use are those calculated in Davey et al. (1968, p. 39, Table 2). We remark that in order to calculate such coefficients, large amounts of algebra and numerical solution of non-homogeneous linear problems are necessary.

Since, as $k \rightarrow 0$

$$
h_{0} \rightarrow f_{0}, \quad h_{20} \rightarrow f_{20}, \quad h_{30} \rightarrow f_{30},
$$

by comparing terms of the same order in the higher-order equations we find that

$$
a_{4}=2 a_{1}, \quad b_{1}=3 a_{1} .
$$

Using these approximations we obtain several families of steady solutions.

\subsection{Possible flow patterns}

We list the steady or uniformly rotating/translating flow patterns that can arise as solutions of the Navier-Stokes equations near criticality. By an axial translation, we can assume that $A_{s}=0$ for all of the solutions listed below.

Couette flow

This corresponds to the trivial solution

$$
A_{c}=B_{c}=B_{s}=0,
$$

and is stable for $T<T_{c}$. 
Taylor vortex flow

This is given by

$$
A_{c}=\left(-a_{0} / a_{1}\right)^{1 / 2}, \quad B_{c}=B_{s}=0,
$$

and is the primary instability on increasing $T$ through $T_{c}$, the critical Taylor number. Ribbons

(Also referred to as the non-axisymmetric simple mode in Davey et al. 1968.) Although it is observed to always be unstable in Davey et al. (1968) there can be a steady flow

$$
B_{c}=\left(-\frac{b_{0 r}}{3 a_{1}}\right)^{1 / 2} \mathrm{e}^{\mathrm{i} \omega \tau}, \quad A_{c}=B_{s}=0,
$$

with $\omega=b_{0 i}$. This is a primary instability of Couette flow.

Wavy vortex mode

Standard Taylor vortex flow can lose stability at higher Taylor numbers to wavy vortices given by

$$
A_{c}=\left[-\left(\frac{3 a_{0}-2 b_{0 r}}{3 a_{1}-2 b_{4 r}}\right)\right]^{1 / 2}, \quad B_{s}=\left[-\left(\frac{b_{0 r}-\left(b_{4 r} a_{0}\right) / a_{1}}{3 a_{1}-2 b_{4 r}}\right)\right]^{1 / 2} \mathrm{e}^{\mathrm{i} \omega \tau}, \quad B_{c}=0,
$$

with $\omega=b_{0 i}-b_{4 i}^{2} A_{c}^{2}$.

Twisted vortices

These non-axisymmetric perturbations of standard Taylor vortices retain the flat in- and outflow boundaries of the latter. They satisfy

$$
A_{c}=\left[-\left(\frac{2 b_{0 r}-a_{0}}{5 a_{1}}\right)\right]^{1 / 2}, \quad B_{c}=\left[-\left(\frac{3 a_{0}-b_{0 r}}{15 a_{1}}\right)\right]^{1 / 2} \mathrm{e}^{\mathrm{i} \omega \tau \tau}, \quad B_{s}=0,
$$

and $\omega=b_{0 i}$. These are always observed to be unstable for the parameters examined in Davey et al. (1968).

Spiral mode

Although spirals are not stable for the parameters investigated in Davey et al. (1968), they can be stable at other parameter values. They are given by

$$
B_{c}=\left(-\frac{b_{0 r}}{4 a_{1}}\right)^{1 / 2} \mathrm{e}^{\mathrm{i} \omega \tau}, \quad B_{s}=\mathrm{i} B_{c}, \quad A_{c}=0,
$$

with $\omega=b_{0 i}$. This is a primary instability of Couette flow.

Note that all of the above modes can be experimentally or numerically found on varying the angular velocities of both inner and outer cylinders (see Andereck et al. 1986). In particular, twisted vortices have been reported ('ordinary twist solution') in a system of co-rotating cylinders by Weisshaar, Busse \& Nagata (1991) and ribbons in a system of counter-rotating cylinders by Tagg et al. (1989). The parameters where the Davey et al. asymptotic model is valid only display stable Couette, Taylor vortex or wavy vortex flow.

\section{Observed particle paths and flow structure}

Substituting the expressions for $(u, v, w)$ from (2.5) into (2.3) and then integrating the equations with respect to time gives approximate particle paths for the flows. Table 1 gives some example amplitudes of the various modes used for constructing 


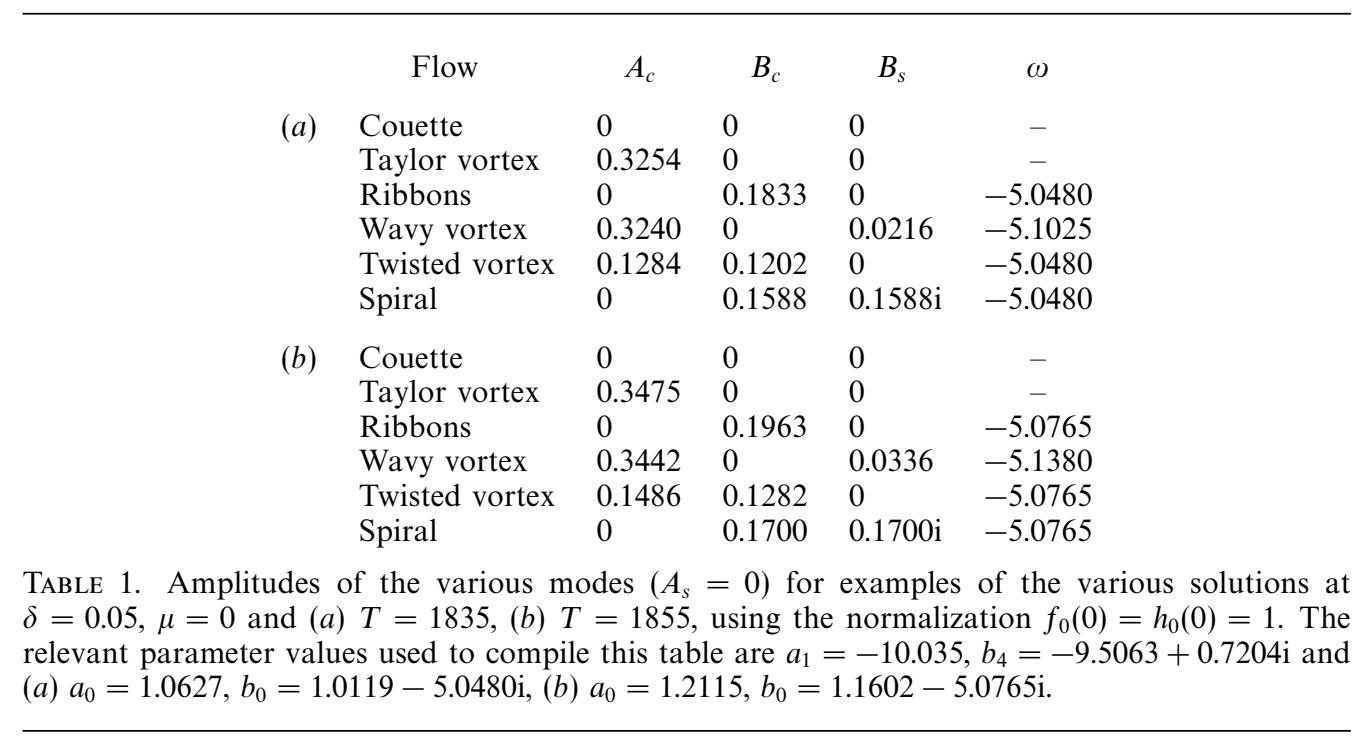

the vector fields when the Taylor number is fixed to be $(a) T=1835,(b) T=1855$. In both cases, $\delta=0.05$ and $\mu=0$. Only instability of the azimuthal mode $m=1$ is considered. The values for $T=1855$ are used for the remainder of this section.

In order to understand the mixing properties seen in simulations, we show flow skeletons of the investigated flows. These are 'cartoons' of the flow showing stagnation points, periodic particle paths and the stable and unstable manifolds for the velocity field

$$
\left(U_{R}, U_{\psi}, U_{Z}\right)=\left(u_{R}, u_{\psi}, u_{Z}\right) / f(x)
$$

with $f(x)$ a function chosen to remove the singularity on the boundaries caused by the no-slip conditions: $f(x)>0$ for $|x|<0.5$ and $f(x)=0$ on $x= \pm 0.5$. The velocity field on the boundary we refer to as the skin friction field. The lines marked $r_{i}$ are periodic trajectories of the skin friction field, $s_{i}$ are periodic trajectories in the fluid while the points $p_{i}$ are stagnation points within the fluid. The inflow and outflow boundaries are near $z=k \pi, k \in \mathbf{Z}$. They are invariant except for wavy vortices and spirals. The figures summarize information gained by time-stepping near the boundaries and searching for fixed points of the velocity field. The flow skeletons were found to be much more informative than plots of the velocity fields. MacKay (1994) has shown that such skeletons provide a framework to discuss transport through surfaces bounded by trajectories contained within the flow skeleton. We note that all flow skeletons presented can be thought of as mixtures of the pure modes - Taylor vortex and ribbon flow.

The particle paths were computed with the package dstool of Guckenheimer et al. (1991) using a fourth-order Runge-Kutta integrator with variable stepsize and constant fractional error between $10^{-5}$ and $10^{-7}$. The qualitative features of the observed particle paths are described in the following subsections.

\subsection{Couette flow}

For Couette flow (not illustrated), the particles move at constant $x$ and $z$ around the cylinder in the $\theta$-direction at a rate determined solely by their initial $x$. 


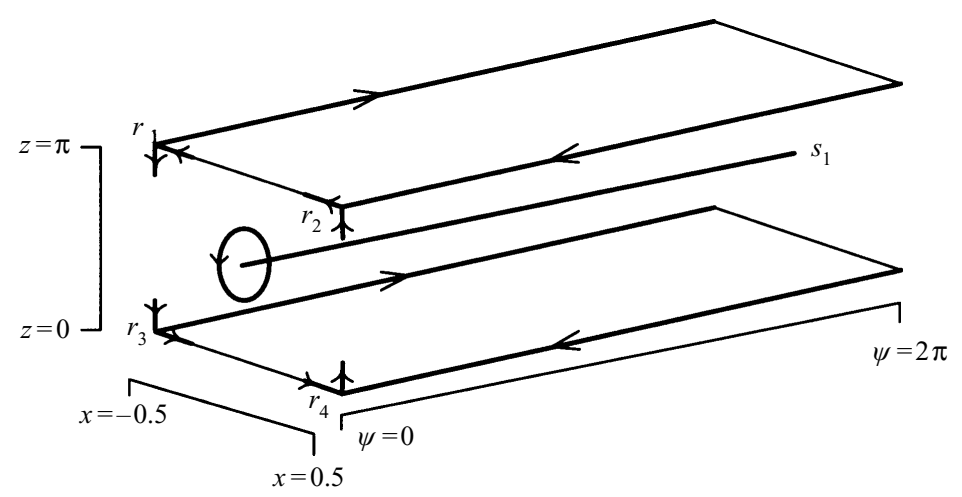

FIGURE 1. A flow skeleton in a co-rotating frame for Taylor vortices. The cylinder has been 'unrolled'; the flow is $2 \pi$-periodic in the $\psi$-direction. There are no stagnation points for almost all rotation speeds, but there are hyperbolic periodic orbits $r_{i}$ for the skin firction field and elliptic periodic orbits $s_{1}$ in the fluid. At a particular co-rotating speed, $s_{1}$ becomes a degenerate line of stagnation points. The orbits $r_{1}$ and $r_{4}$ are repelling in the $z$-direction whereas $r_{2}$ and $r_{3}$ are attracting in this direction. The symmetries $(x, \psi, z) \mapsto(x, \psi, 2 \pi-z)$ and $(x, \psi, z) \mapsto(x, \psi, 2 \pi+z)$ mean that $z=0, \pi$ are invariant planes.

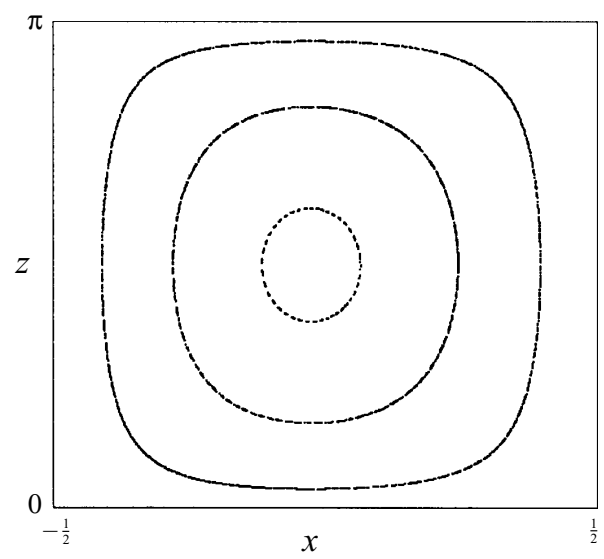

FIGURE 2. Typical trajectories for three initial conditions advected by Taylor vortex flow projected into the $(x, z)$-plane. This and all subsequent figures are calculated with $T=1855, \delta=0.05$ and $\mu=0$ (outer cylinder stationary). There is for each trajectory a uniform average propagation in the azimuthal direction.

\subsection{Taylor vortex flow}

For Taylor vortex flow (flow skeleton in figure 1 and particle paths shown in figure 2 projected into the $x, z$-plane) almost all particle trajectories are dense on the surface of tori. Note that the wave speed is not defined; we take the wave speed for ribbons to allow easy comparison. A typical surface of section shows an integrable map preserving an area form. This integrability can be thought of as resulting from the presence of the one-parameter symmetry group $\mathbf{S O}(2)$ of rotations around the axis of the cylinder. The motion in the $(x, z)$-plane is decoupled from that in the azimuthal direction. 


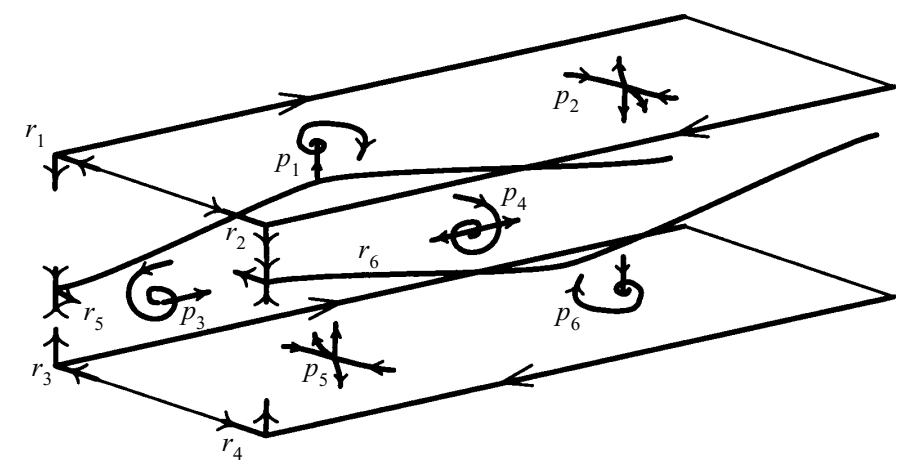

FiguRE 3. Flow skeleton in the co-rotating frame for ribbon flow. The coordinates are as in figure 1. There are invariant planes at $z=k \pi$ and associated periodic orbits for the skin friction field $r_{i}$, $i=1, \cdots, 4$. Since there is a symmetry $(x, \psi, z) \rightarrow(x, \psi+\pi, \pi-z)$ which maps the $r_{1}$ to $r_{3}$ and $r_{2}$ to $r_{4}$, these have the same stability and are observed to be separated by periodic orbits $r_{5}$ and $r_{6}$ of the skin friction field. There are elliptic stagnation points $p_{1}$ and $p_{3}$ and a hyperbolic stagnation point $p_{2}$. These have symmetric images at $p_{6}, p_{4}$ and $p_{5}$.

\subsection{Ribbons}

Figure 3 shows the flow skeleton for ribbons (pure non-axisymmetric mode). Observe that there are periodic orbits on the inner and outer skin friction fields between the inflow and outflow boundaries. The velocity fields are similar to those found by Tagg at al. (1989). There are two-dimensional unstable manifolds from the boundaries into the fluid, and we expect a very complicated pattern of intersection and folding occurring within the fluid. Indeed, for this pure non-axisymmetric mode with $A_{c}=A_{s}=B_{s}=0, B_{c} \neq 0$ there seems to be a large degree of mixing even at small $\left|B_{c}\right|$. Figure $4(a, b)$ shows a single trajectory in two different projections. Figure 4(c) shows the intersections of a trajectory with the surface $x=0$. Figure $4(d)$ shows projection onto the $(\psi, z)$-plane of the stagnation points $p_{3}$ and $p_{4}$ near the centre of the ribbon (in the rotating reference frame). These points have linearized eigenvalues consisting of a complex pair and a real eigenvalue whose one-dimensional manifolds (illustrated) are observed to fill out a large region of the fluid in a complicated manner. Note that one can see ribbon flow as a superposition of spirals of equal amplitude and opposite handedness. The elliptic stagnation points can be thought of as the intersections of the centres of these spirals.

\subsection{Wavy vortex flow}

This flow can be thought of as a perturbation of Taylor vortex flow that breaks the rotational symmetry by imposing a modulation in the $\psi$-direction; we do not expect all particle paths to be integrable. Figure 5 shows this clearly; the two-dimensional unstable manifold of $r_{2}$ will intersect the stable manifold of $r_{1}$ transversely giving rise to a homoclinic orbit and thus chaotic behaviour.

Figure 6(a) shows a trajectory of a particle on a streamtube that is a deformation of one for Taylor vortex flow. Figure $6(b)$ shows another trajectory that propagates chaotically up and down in the axial direction.

As discussed in Ashwin et al. (1995) a small slowing of the wave velocity can cause the appearance of ring vortices propagating azimuthally around the axis and two stagnation points near the centre of the vortex. Numerical studies of Marcus $(1984 a, b)$ indicate that there should be stagnation points at the vortex centre in a co-rotating frame of reference, and only on slowing the wave speed by a factor of 
(a)

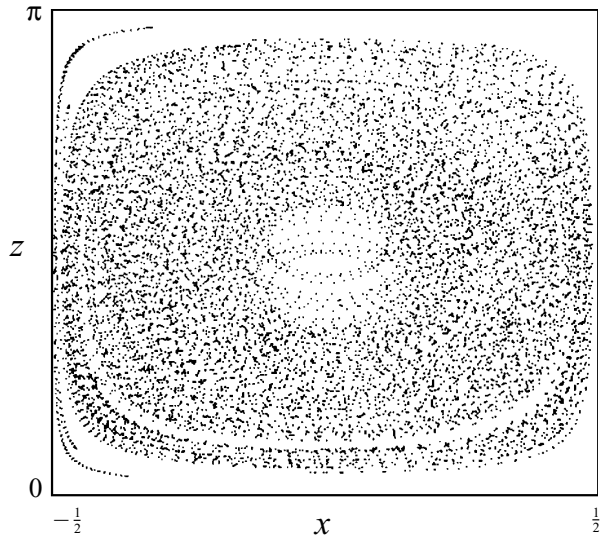

(c)

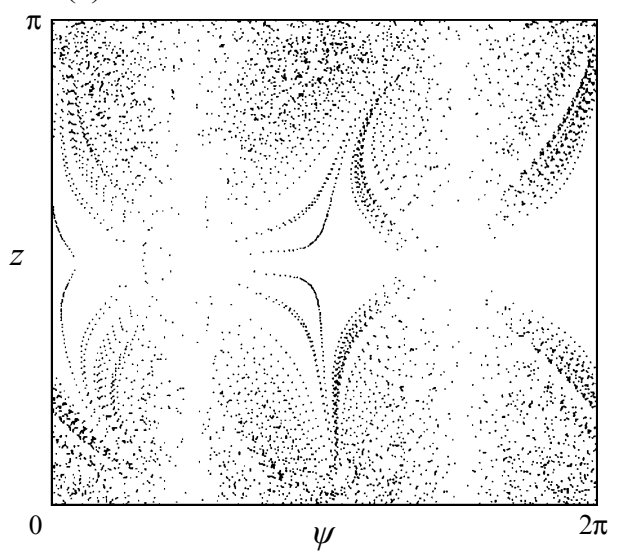

(b)

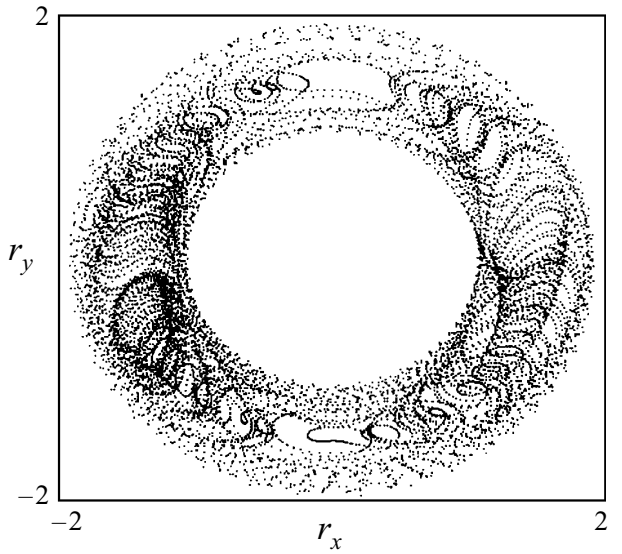

$(d)$

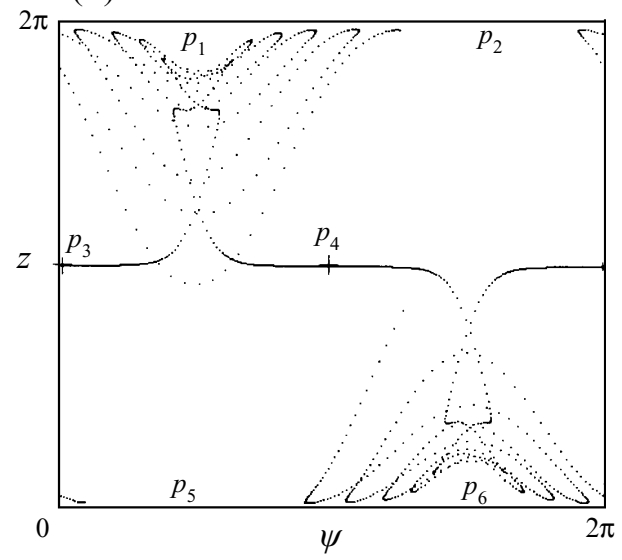

Figure 4. Projections of the trajectory of a single particle in ribbon flow $(a)$ in the $(x, z)$-plane, and $(b)$ the same trajectory in the rotating $\left(r_{x}, r_{y}\right)$-plane. $(c)$ Shows intersections of a trajectory with the plane $x=0$. Note that it appears to fill out a large proportion of the fluid volume. (d) Two stagnation points at the vortex centre near $z=\pi / 2, x=0$ are at $p_{3}$ and $p_{4}$ (cf. figure 3 ) and parts of the one-dimensional manifolds from these stagnation points are shown. They are forced away from the centerline by the unstable manifolds of stagnation points $p_{5}$ and $p_{2}$ towards the spiral stagnation points $p_{1}$ and $p_{6}$.

about $1 \%$ can we achieve this. This is supported by evidence presented in Jones (1985) that an effect of higher-order terms is to slow the wave speed. The possible existence of these 'coherent structures' was reported in Ashwin, Mann \& King (1995). We discuss the effect the chaotic behaviour near the edges of the vortex has on axial transport in $\S 5$.

One such pair of ring vortices is shown in figure 7 where the wave speed has been set at $\omega=5.085$. We show only intersections of the particle trajectories with the plane $x=-0.03$ and trajectories of three initial conditions are shown. As noted by Broomhead \& Ryrie (1988) for the case of travelling waves, there does not seem to be a Poincare section that intersects all particle paths. This contrasts with Ashwin et al. (1995) where there is a fixed section that intersects all particle paths for small enough eccentricities. Between regions of integrable behaviour there is a densely filled region of chaotic behaviour where mixing between vortices takes place. In this investigation 


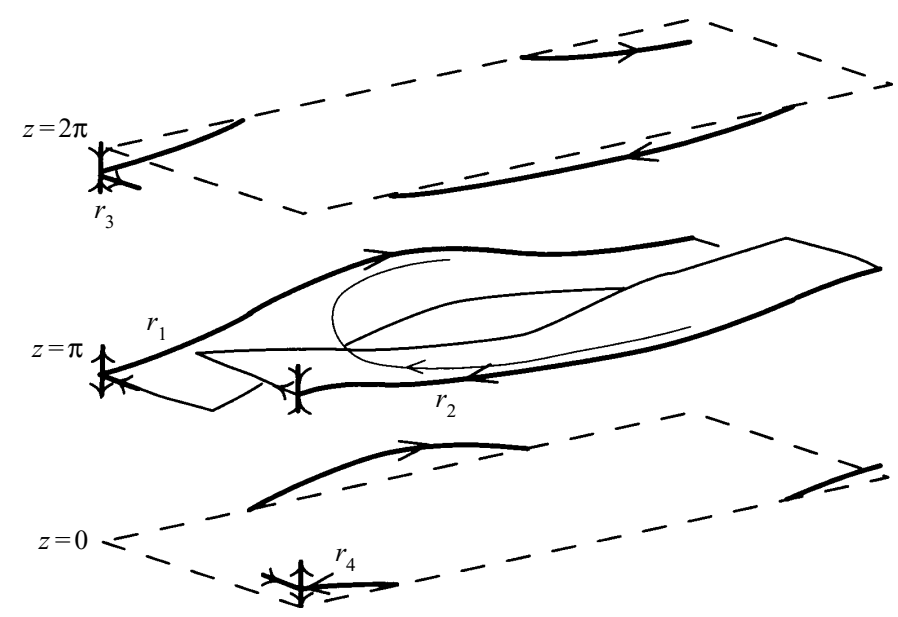

FiguRE 5. Flow skeleton in the co-rotating frame for wavy vortex flow. The coordinates are as in figure 1 , but a whole period of $z$ is necessary to specify the flow. The symmetry-forced invariant planes of Taylor vortex flow are absent, and the associated periodic orbits of the skin friction field $r_{i}, i=1, \cdots, 4$ are perturbed. An orbit connecting $r_{2}$ to $r_{1}$ and parts of their two-dimensional unstable, resp. stable manifolds are illustrated. At the centre of the wavy vortex (not illustrated) depending on the wave speed there is either an elliptic periodic orbit or stagnation points. This flow can be thought of as Taylor vortex flow (figure 1) perturbed by low-amplitude ribbon flow (figure 3 ) translated by $\pi / 2$ in the $z$-direction.

(a)

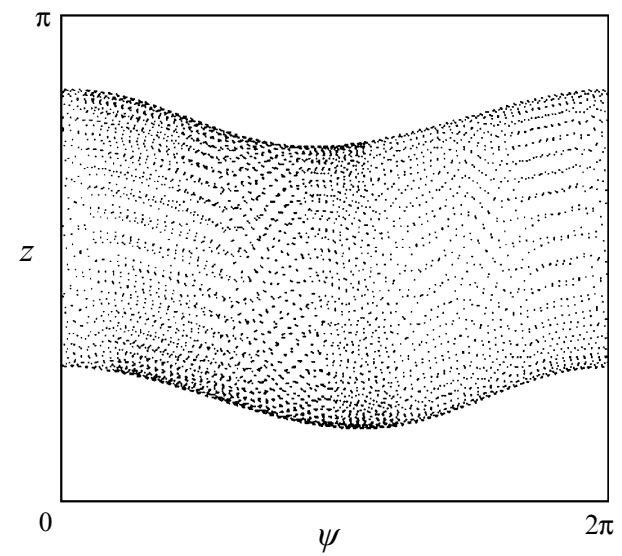

(b)

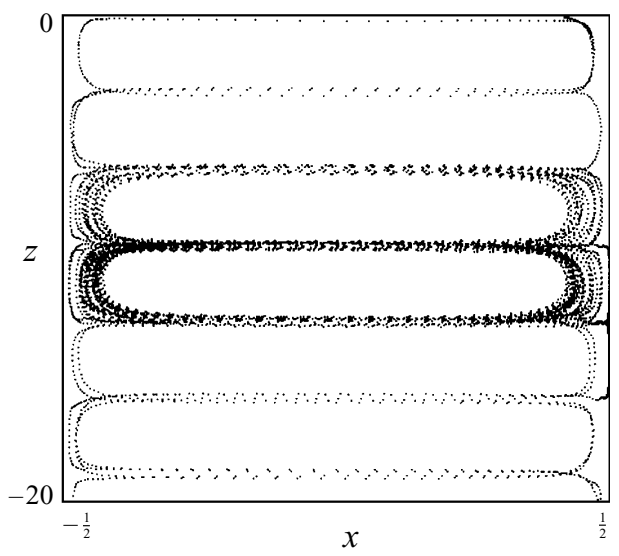

FiguRE 6. Wavy vortex flow. (a) The trajectory of a particle that remains on the surface of a two dimensional torus which is a deformation of one corresponding to Taylor vortex flow. $(b)$ A trajectory of a particle near the edge of the vortex that moves up and down the cylinder in a seemingly random manner. This is shown in the $(x, z)$-plane (note the exaggerated axial scale).

there seemed to be a region of almost totally integrable behaviour separated from a region of almost totally chaotic behaviour (no observable 'islands' were found) by a boundary that could be non-smooth. This is unlike the picture obtained by Broomhead \& Ryrie (1988) and also unlike that expected for small perturbations of integrable twist maps. Whether this is significant is a question for further study.

The dependence of the wave speed of wavy vortex flow on system parameters has been a subject of intense study (see e.g. King et al. 1984). Some careful numerical 


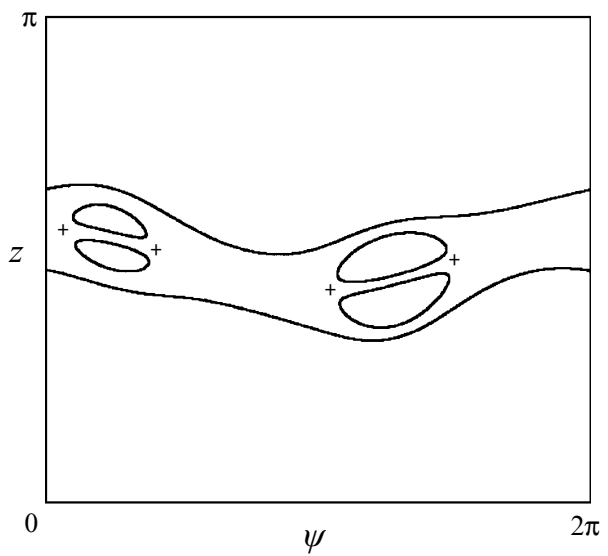

FIGURE 7. Intersection of three trajectories with a surface of section $x=-0.03$ for wavy vortex flow with the wave speed slowed to give stagnation points in the rotating frame of reference. Note the presence of two ring vortices and a deformed Taylor vortex. The interior stagnation points are marked by + signs. Note that there is no exact symmetry that permutes the two ring vortices.

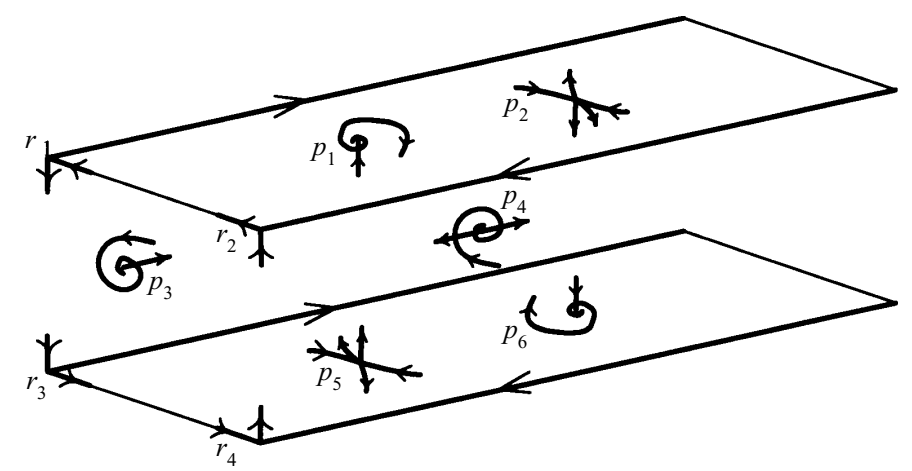

FiguRE 8. Flow skeleton in the co-rotating frame for twisted vortex flow. The coordinates are as in figure 1. There are symmetry-forced invariant planes but unlike ribbon flow, the stabilities of $r_{2}$ and $r_{4}$ are different and there are no periodic orbits of the skin friction field corresponding to $r_{5}$ and $r_{6}$. This flow can be seen as a mixture of the ribbon and the Taylor vortex mode. Note that increasing the relative amplitude of the Taylor vortex mode will cause the pairs of stagnation points $p_{1}, p_{2}$ and $p_{5}, p_{6}$ to annihilate each other in saddle-node bifurcations.

simulations have been carried out by Marcus (1984a,b) and Jones (1985) and we have compared our flow field with these. Because we are much closer to onset of waves and do not include the nonlinear effects taken account of by Marcus, the distortion of the co-moving surface in our velocity field is much smaller than that found by Marcus (1984b).

The most noticeable feature on examining projections of the velocity field of wavy vortices is that there is little obvious sign of the propagating ring vortices! This is due to the fact that the main motion on the ring vortex consists of circulation around the centre of the vortex on streamlines that almost close up. Only over much longer timescales (not visible on the resolution of the velocity field plots) does the drifting effect appear. Note that $R=1.04 R_{c}$ for our model whereas $R=2.063 R_{c}$ for Marcus' calculations; moreover, we work with a very small gap apparatus. Thus, we expect 
(a)

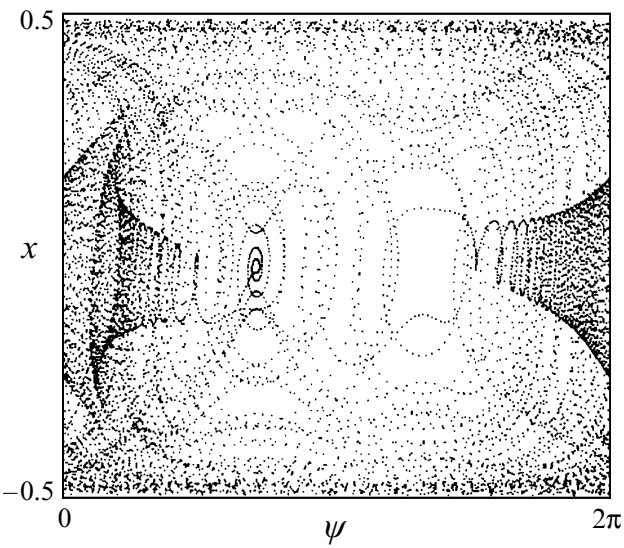

(b)

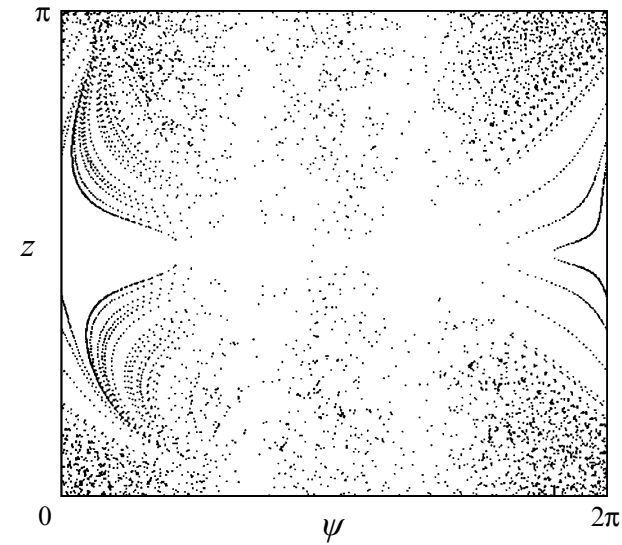

FiguRE 9. (a) Typical trajectory within a twisted vortex in the $(\psi, x)$-plane. $(b)$ The intersection of a single trajectory in this flow with the plane $x=0$. There appears to be a large region of mixing.

his calculations will have more energy in high-order harmonics of the fundamental instability.

\subsection{Twisted vortex mode}

This steadily rotating solution of the Navier-Stokes equations has inflow and outflow boundaries that form barriers to the vertical diffusion of fluid particles. Figure 8 shows the flow skeleton for such a flow; note that there are qualitative features from Taylor vortices in addition to those from ribbon flow. Furthermore there is no symmetry operation that interchanges the stagnation points $p_{3}$ and $p_{4}$, unlike for ribbon flow.

Figure 9 shows a typical trajectory in twisted vortex flow. As with ribbons, and in contrast to wavy vortices, there appear to be large regions of chaotic mixing. We attribute this to the fact that the relative amplitude of the non-axisymmetric perturbation to the symmetric flow is much higher for twisted vortex than for wavy vortex flow (see table 1).

\subsection{Spiral flow}

The presence of a continuous symmetry of the flow pattern forces this flow to be integrable. The continuous symmetry acts by

$$
(x, \psi, z) \rightarrow(x, \psi+s, z-s)
$$

where $s \in \mathbf{R}$. This corresponds to a rotation in the azimuthal direction coupled with a translation in the axial direction. Figure 10 shows a typical particle trajectory in this flow; all particles propagate in the axial direction with a uniform asymptotic speed. The speed and direction of this propagation is dependent on the initial position.

Spiral flow is the only flow that breaks the symmetry of reflection of the flow pattern in all planes perpendicular to the axis. Thus an assumption of a periodic velocity field does not imply that the pressure field is necessarily periodic (see $\$ 5$ for a further discussion of this).

Note that all of the above flow patterns are asymptotic solutions to the NavierStokes equations at the same parameter values. However, only the wavy vortex flow is 
(a)

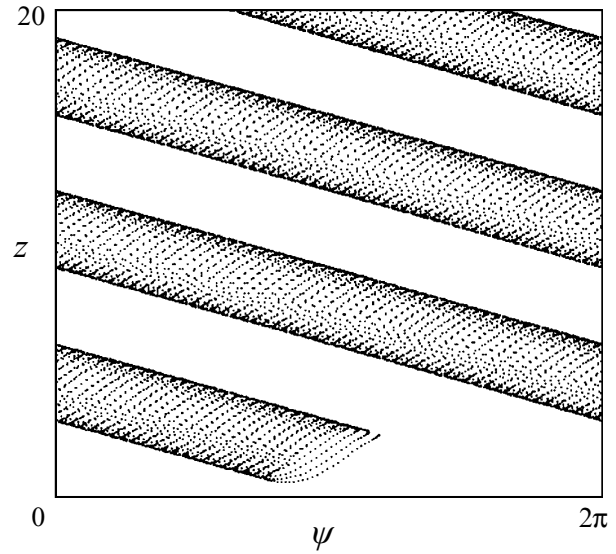

(b)

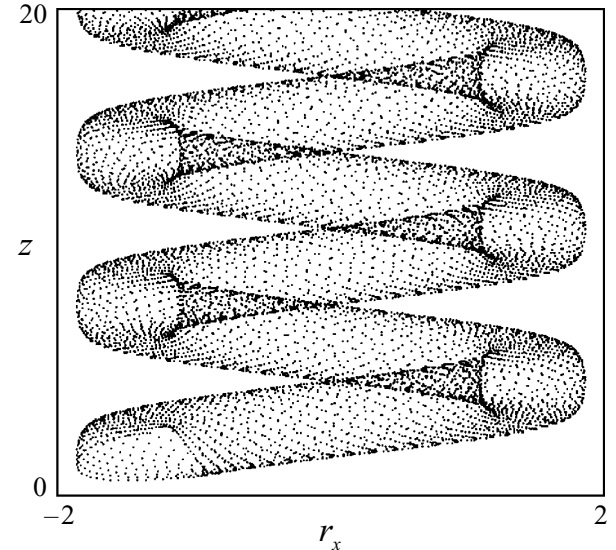

FIGURE 10. A single trajectory within spiral flow shown $(a)$ in the $(z, \psi)$-plane and $(b)$ in the $\left(r_{x}, z\right)$-plane. This particle shows a slow drift towards positive $z$.

(a)

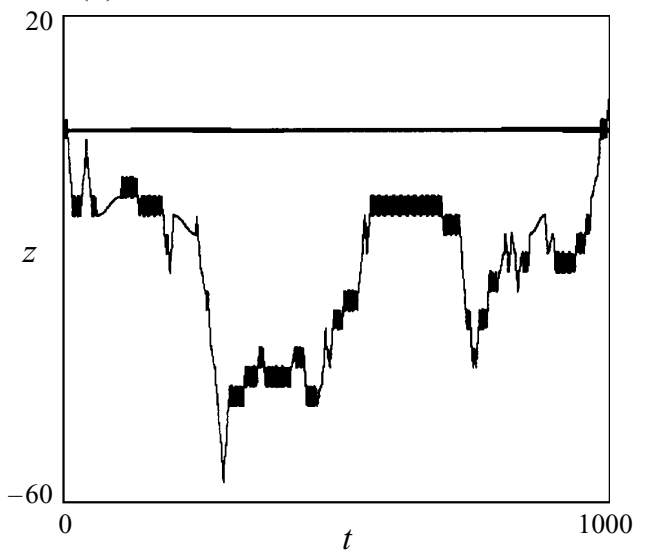

(b)

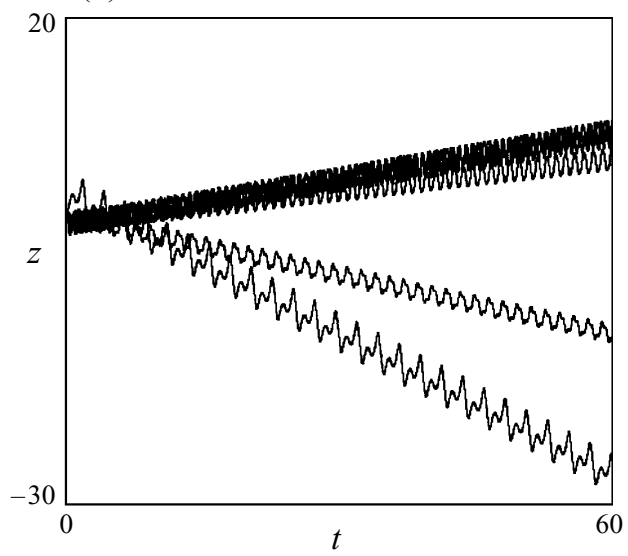

FiguRE 11. Motion in the axial direction of $(a)$ two particles in the axial direction under the influence of wavy vortex flow and $(b)$ six particles under the influence of spiral flow. Observe that for $(a)$ one particle (started near the boundary of the vortices) appears to follow a random walk over large timescales whereas the other (started within a ring vortex) remains bounded within one vortex. In $(b)$, each particle shows uniform average propagation in the axial direction at a rate that is dependent on the initial condition. Particles near the edge of the spiral (with multiple local maxima) propagate downwards whereas those near the centre propagate upwards.

dynamically stable according to the calculations of Davey et al. (1968). We remark that the integrability of the spiral and Taylor vortex flows can be inferred as an application of results of Mezic \& Wiggins (1994b).

\section{Discussion and conclusions}

\subsection{Axial transport}

As a measure of the speed of mixing between vortices in the $z$-direction, one can define an average diffusion rate by averaging over an ensemble of particles (see e.g. Broomhead \& Ryrie 1988; Hydon 1994). In practice it is difficult to say how useful 

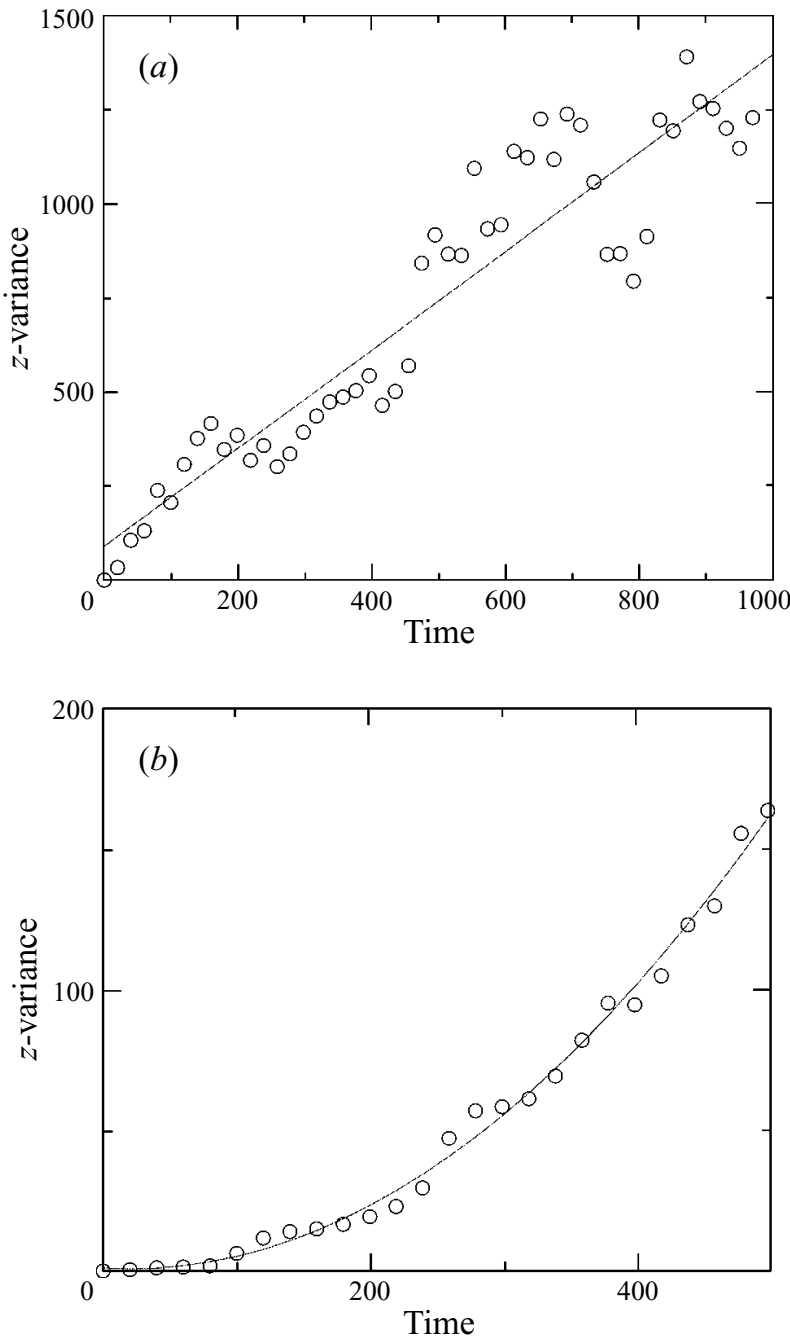

FiguRE 12. Temporal evolution of axial variance of ten particles started near the outside cylinder. (a) Wavy vortex flow; this shows a typical linear growth expected in a well-mixed layer. The growth rate is approximately 1.28 (scaled) $z$-units per time unit. (b) Spiral flow; this has quadratic growth of the variance as expected for a non-chaotic laminar flow.

the measurements from this are due to the effect of, for example, periodic orbits that propagate at non-zero mean axial velocity.

As a simple demonstration of the appearance of axial transport, figure 11 shows time series of $z$ for example test particles in $(a)$ wavy vortex flow and $(b)$ spiral flow. Note that $(a)$ shows a slow random-walk-like behaviour in the axial direction whereas (b) shows relatively fast deterministic dispersion of particles, with variance scaling as $t^{2}$ (see e.g. Mezic \& Wiggins 1994a). Figure 12 shows the corresponding change in the axial variance of a group of ten particles started near the outer boundary for the the flows in figure 11. Note the growth in variance in $(a)$ is linear in time indicating a well-mixed diffusion-like chaotic process; that in $(b)$ grows quadratically as is to be expected for integrable non-chaotic flow. In particular in the chaotic region of wavy vortex flow any anomalous diffusion behaviour appears to be quite weak, indicating 
that cantori 'barriers' to the flow cause relatively little trapping in the mixing layer between the vortices.

Axial transport in Taylor vortex and spiral flow has been experimentally and numerically examined by F. Marquès, D. Crespo \& J. Sánchez (personal communication): they identified fundamentally different mechanisms of transport: diffusion driven for Taylor vortices and advection driven for spiral flow. We remark that Sagues \& Horsthemke (1986) have investigated axial transport for Taylor vortices under the addition of small noisy perturbations.

\subsection{Integrability of spiral flow}

We now demonstrate explicitly how spiral flow can be viewed as integrable. We assume that the azimuthal wavenumber of the spirals is $m=1$. If we set

$$
\begin{aligned}
& \Psi=\omega t+\theta+z, \\
& \Xi=\omega t+\theta-z,
\end{aligned}
$$

then for $B_{s}=\mathrm{i} B_{c}=\mathrm{i} B$ the equations for particle paths (2.3) (with time scaled by a factor of $\alpha$ ) can be written

$$
\begin{aligned}
& \dot{x}=-B h_{20}(x) \mathrm{e}^{\mathrm{i}(z+\theta+\omega t)}+\text { c.c. } \\
& \dot{\theta}=\alpha \frac{(T \delta)^{1 / 2}}{2}\left(1-\alpha x+B h_{0}(x) \mathrm{e}^{\mathrm{i}(z+m \theta+\omega t)}\right)+\text { c.c. }, \\
& \dot{z}=-B\left(\mathrm{i} D h_{20}(x)+k \alpha h_{0}(x)\right) \mathrm{e}^{\mathrm{i}(z+\theta+\omega t)}+\text { c.c. }
\end{aligned}
$$

and so

$$
\begin{aligned}
& \dot{x}=-B h_{20}(x) \mathrm{e}^{\mathrm{i} \Psi}+\text { c.c. } \\
& \dot{\theta}=\alpha \frac{(T \delta)^{1 / 2}}{2}\left(1-\alpha x+B h_{0}(x) \mathrm{e}^{\mathrm{i} \Psi}\right)+\text { c.c. } \\
& \dot{z}=-B\left(\mathrm{i} D h_{20}(x)+k \alpha h_{0}(x)\right) \mathrm{e}^{\mathrm{i} \Psi}+\text { c.c. }
\end{aligned}
$$

In the new coordinates this gives

$$
\begin{aligned}
\dot{x} & =-B h_{20}(x) \mathrm{e}^{\mathrm{i} \Psi}+\text { c.c. }, \\
\dot{\Psi} & =\omega+\alpha k(1-\alpha x)-\mathrm{i} B D h_{20}(x) \mathrm{e}^{\mathrm{i} \Psi}+\text { c.c. }, \\
\dot{\Xi} & =\omega+\alpha k(1-\alpha x)+B\left(2 \alpha k h_{0}(x)+\mathrm{i} D h_{20}(x)\right) \mathrm{e}^{\mathrm{i} \Psi}+\text { c.c. }
\end{aligned}
$$

Note that the equations for $x$ and $\Psi$ decouple from the $\Xi$ variable: this is a manifestation of the integrability of the flow. The equations for $x$ and $\Psi$ can be written in Hamiltonian form by defining the conserved quantity

$$
H(x, \Psi)=\omega x+\alpha k\left(x-\alpha \frac{x^{2}}{2}\right)-\mathrm{i} B h_{20}(x) \mathrm{e}^{\mathrm{i} \Psi}+\text { c.c. }
$$

and noting that

$$
\dot{x}=-\frac{\partial H}{\partial \Psi}, \quad \dot{\Psi}=\frac{\partial H}{\partial x} .
$$

Given an approximation for $h_{20}(x)$ it is possible to calculate the level curves of $H$ and thereby the motion in the $(x, \Psi)$-plane. Note that the $\Psi$-independent terms mean that the two counter-rotating spirals are not symmetrically related. One can infer that the centres of the spiral vortices will be arranged so that one of them is closer to the inner cylinder than the other. Such a reduction can be formulated for abstract spiral flows in terms of the vector potential: see Marquès, Crespo \& Sánchez (1995). 


\subsubsection{Mean axial flow for spirals}

It was noted by Edwards et al. (1991) and Raffaï \& Laure (1993) that spiral flows can have non-periodic axial pressure fields. In general, only the pressure gradient must be periodic if the flow is periodic. The ansatz of Davey et al. (1968) is that the velocity field is periodic. However, mean axial flow is a second-order effect, as pointed out in Davey et al. (1968) and confirmed in numerical calculations of Marquès et al. (1995).

The mean axial flow $F$ through the surface $z=z_{0}$ for the first-order approximation can be easily calculated. For fixed $z_{0}$ and $t_{0}$ let

$$
\begin{aligned}
F & =\int_{z=z_{0}, t=t_{0}} \dot{\mathrm{d}} \mathrm{d} x \mathrm{~d} \theta \\
& =-\frac{1}{2} \int_{x=-1 / 2}^{1 / 2} \int_{\Psi=0}^{2 \pi}\left(-B\left(\mathrm{iD} h_{20}(x)+k \alpha h_{0}(x)\right) \mathrm{e}^{\mathrm{i} \Psi}+\text { c.c. }\right) \mathrm{d} x \mathrm{~d} \Psi \\
& =0 .
\end{aligned}
$$

Higher-order corrections are expected to cause non-zero mean axial flow in spirals.

\subsection{Conclusions}

We have performed particle tracking experiments for several different steadily rotating flow patterns in Taylor-Couette flow using the Davey et al. (1968) asymptotic model. It is possible that the ring vortex structures and/or chaotic layers may disappear on adding further terms in the truncation (E. Knobloch, personal communication). However, the observed Lagrangian properties of wavy vortices in $\$ 4.4$ suggest to us that the same sort of mechanisms may be at work in the cores of Taylor vortices at the wavy instability as are found in the classically studied problems of vortex breakdown such as discussed in Leibovich's (1978) review or the series of papers by Lopez (1990), Brown \& Lopez (1990) and Lopez \& Perry (1992). The parallels are as follows:

(a) Recirculating ring vortices aligned with the vorticity are created; these can appear on smoothly moving along a path of steady solutions of the Navier-Stokes equations and do not require any instability or bifurcation of the Eulerian flow.

(b) The closeness of the central region to integrability suggests that this is fundamentally an axisymmetric phenomenon as in the first stage of vortex breakdown; it suggests that it is caused by inherent axisymmetric instability of the vortex core rather than pure deformation of the outer regions of the vortex.

(c) The breakdown of steady vortex instability to a time-periodic flow happens in both cases, rather than breakdown via a secondary steady instability.

Although all the non-axisymmetric flow patterns except for wavy vortex flow are unstable at the parameter values investigated, the particle paths for unstable flows are nevertheless interesting on two counts. Firstly, there are parameter values where all of the studied flow patterns are observed to be stable, and this provides a guide to possible particle behaviour in these cases. Secondly, in turbulent flow the pattern may linger near unstable steady flow patterns of saddle type (H. K. Moffatt, personal communication); thus the particle paths for unstable flow patterns will be important in describing behaviour of particles in unsteady flows.

Because the Broomhead \& Ryrie (1988) model for particle motion in wavy vortex flow only considers an azimuthal symmetry-breaking perturbation in the $z$-component of the velocity field, there is no possibility of their model picking up the presence of coherent structures that are confined in the azimuthal direction. The model of Davey 
et al. (1968) has components in all directions and is dependent on all variables. It has the great advantage that it is truly (asymptotically) a solution of the Navier-Stokes equations. The qualitative appearance of mixing between vortices in wavy vortex flow is very similar; of course, this depends on the aspect ratio as well as the Taylor number and there is no rigorous way to connect these parameters with the parameter $\epsilon$ used by Broomhead \& Ryrie.

As this study has been based on an asymptotic solution of the Navier-Stokes equations, and since this solution necessarily increases the regions of chaotic advection as it loses validity, it should be stressed that this is still only a guide as to what might happen. The results of Ashwin \& King (1995) on a similar asymptotic model for Taylor vortices in an eccentric system show that on including higher-order terms in the asymptotic expansion the pattern of chaotic mixing was only perturbed to a small extent and this lends support to the predictions from that model. However, in this paper there are additional approximations. Firstly, we assume that just the Taylor vortex and $m=1$ modes are important for the dynamics. Secondly we assume the modes with identical axial wavenumber are the only important ones. Thirdly we assume that certain third-order coefficients can be approximated by their axisymmetric counterparts.

By performing particle tracking experiments for more accurate flow fields derived from numerical approximations we hope that we should be able to shed more light on mixing effects in such flows. Additionally, there are possibilities for experimental work to locate possible ring vortex structures and compare with mixing in experimental realizations of such flows. Theoretically, there is work to be done to clarify the reason for the large regions of chaotic mixing for ribbons and twisted vortices.

We have investigated diagrams of vorticity and velocity fields, but up to now have not been able to identify, for example, regions of large mixing. This is presumably because we are not near the limit of zero viscosity (Euler flows) where, for example, trajectories are confined to surfaces of constant helicity. What is more revealing about the mixing behaviour than plots of velocity and vorticity is the identification of the stagnation points, their stable and unstable manifolds and other features of the flow skeleton. Drawing together the present study and the eccentric Taylor vortex model (Ashwin \& King 1995) we find the following.

Two integrable flows: Taylor vortices and spirals. The integrable behaviour is due to symmetry constraints.

Three flows with a high degree of mixing throughout a vortex: ribbons, twisted vortices, eccentric Taylor vortex with separation. The high degree of mixing is related to the presence of stagnation points and periodic orbits whose stable and unstable manifolds intersect in a complicated manner in the flow interior. Is there a link between the instability of ribbons and twisted vortex flow, and their high degree of mixing?

Two flows where near-integrable behaviour exists in the flow interior and mixing is confined to a layer adjacent to the vortex boundary: wavy vortex flow, eccentric Taylor vortex (small eccentricity). It has been suggested (I. Mezić, personal communication) that the near-integrable core may be due to the flow there being close to an Euler flow; for Euler flows the trajectories are confined to surfaces of constant helicity.

One flow with inter-vortex mixing: wavy vortex flow. In Taylor vortex flow the vortices are separated by invariant surfaces. The onset of wavy vortex flow breaks this connection and this gives rise to inter-vortex mixing.

Future work needs to address the question of what happens as the Reynolds number is increased. This cannot be done with the perturbative models we have studied thus 
far. It must be done with fully nonlinear solutions obtained from simulations of the Navier-Stokes equations. It should then be possible to begin to draw some general conclusions about the physics of chaotic advection.

The research of PA and GPK was partially supported by the EPSRC under the Nonlinear Initiative. PA thanks the European Union for support with HCM fellowship ERBCHBCT930503 while completing this research. We thank the following people for very helpful conversations: Tom Bridges, Pascal Chossat, Peter Hydon, Patrice Laure, Igor Mezić and George Rowlands.

\section{REFERENCES}

Andereck, C. D., LiU, S. S. \& Swinney, H. L. 1986 Flow regimes in a circular Couette system with independently rotating cylinders. J. Fluid Mech. 164, 155-183.

Ashwin, P. \& King, G. P. 1995 Streamline topology in eccentric Taylor vortex flow J. Fluid Mech. 285, 215-247.

Ashwin, P., Mann, G. W. \& King, G. P. 1995 Azimuthally propagating ring vortices in a model for nonaxisymmetric Taylor vortex flow. Phys. Rev. Lett. 75, 4610-4613.

Broomhead, D. S. \& Ryrie, S. C. 1988 Particle Paths in wavy vortices Nonlinearity 1, 409-434.

Brown, G. L. \& Lopez, J. M. 1990 Axisymmetric vortex breakdown Part 2. Physical mechanisms. J. Fluid Mech. 221, 553-576.

Chossat, P. \& Iooss, G. 1994 The Couette-Taylor Problem. Springer.

Davey, A., DiPrima, R. C. \& Stuart, J. T. 1968 On the instability of Taylor vortices. J. Fluid Mech. 31, 17-52.

DiPrima, R. C. \& Stuart, J. T. 1975 The nonlinear calculation of Taylor-vortex flow between eccentric rotating cylinders. J. Fluid Mech. 67, 85-111.

Edwards, W. S., Tagg, R. P., Dornblaser, B. C. \& Swinney, H. L. 1991 Periodic travelling waves with nonperiodic pressure. Eur. J. Mech. B/Fluids 10 (2, suppl.), 205-210.

Golubitsky, M., Stewart, I. N. \& SchaefFer, D. 1988 Groups and Singularities in Bifurcation Theory, Vol. 2. Springer.

Guckenheimer, J., Myers, M. R., Wicklin, F. J. \& Worfolk, P. A. 1991 Dstool: a Dynamical Systems Toolkit with an Interactive Graphical Interface, User's Manual. Center for Applied Mathematics, Cornell University.

Hydon, P. E. 1994 Resonant advection by oscillator flow in a curved pipe. Physica D 76, 44-54.

Jones, C. A. 1985 The transition to wavy Taylor vortices. J. Fluid Mech. 157, 135-162.

King, G. P., LI, Y., Lee, W., Swinney, H. \& Marcus, P. S. 1984 Wave speeds in wavy Taylor vortex flow. J. Fluid Mech. 141, 365-390.

Krueger, E. R., Gross, A. G. \& DiPrima, R. C. 1966 On the relative importance of Taylor-vortex and non-axisymmetric modes in flow between rotating cylinders. J. Fluid Mech. 24, 521-538.

Leibovich, S. 1978 The structure of vortex breakdown. Ann. Rev. Fluid. Mech. 10, 221-246.

Lopez, J. M. 1990 Axisymmetric vortex breakdown Part 1. confined swirling flow. J. Fluid Mech. 221, 533-552.

Lopez, J. M. \& Perry, A. D. 1992 Axisymmetric vortex breakdown Part 3. Onset of periodic flow and chaotic advection. J. Fluid Mech. 234, 449-471.

MaCKAY, R. S. 1994 Transport in 3D volume-preserving flows. J. Nonlinear Sci. 4, 329-354.

Marcus, P. S. 1984a Simulation of Taylor-Couette flow. Part 1. Numerical methods and comparison with experiment. J. Fluid Mech. 146, 45-64.

Marcus, P. S. $1984 b$ Simulation of Taylor-Couette flow. Part 2. Numerical results for wavy-vortex flow with one travelling wave. J. Fluid Mech. 146, 65-113.

Marquès, F., Crespo, D. \& SÁnchez, J. 1995 Mixing in stratified Couette flow. In Mixing in Geophysical Flows (ed. J. M. Redondo \& O. Métais). CIMNE, Barcelona.

Mezić, I. \& Wiggins, S. 1994a On the dynamical origin of asymptotic $t^{2}$ dispersion of a nondiffusive tracer in incompressible laminar flows. Phys. Fluids 6, 2227-2229.

Mezić, I. \& Wiggins, S. $1994 b$ On the integrability and perturbation of three-dimensional fluid flows with symmetry. J. Nonlinear Sci. 4, 157-194. 
Ottino, J. M. 1989 The Kinematics of Mixing; Stretching, Chaos and Transport. Cambridge University Press.

Perry, A. E. \& ChOng, M. S. 1987 A description of eddying motions and flow patterns using critical point concepts. Ann. Rev. Fluid. Mech 19, 125-155.

RAFFAï, R. \& LAURE, P. 1993 The influence of an axial mean flow on the Couette-Taylor problem. Eur. J. Mech. B/Fluids 12, 277-288.

Sagues, F. \& Horsthemke, W. 1986 Diffusive transport in spatially periodic hydrodynamic flows. Phys Rev A 34, 4136-4143.

Tagg, R., Edwards, S., Swinney, H. L. \& Marcus, P. S. 1989 Nonlinear standing waves in Taylor-Couette flow. Phys. Rev. A 39, 3734-3737.

Weisshaar, E., Busse, F. \& Nagata, M. 1991 Twist vortices and their instabilities in the TaylorCouette system. J. Fluid. Mech. 226, 549-564. 